\title{
DER ISLAM
}

\section{ZEITSCHRIF'T \\ FÜR GESCHICHTE UND KULTUR \\ DES ISLAMISCHEN ORIENTS}

HERAUSGEGEBEN VON

\section{H. BECKER IN BERLIN \\ UND \\ R. STROTHMANN IN HAMBURG}

MIT UNTERSTÜTZUNG DER
HAMBURGISCHEN WISSEN-
SCHAFTLICHEN STIFTUNG

FACHZEITSCHRIFT DER

DEUTSCHEN MORGENLÄNDISCHEN

GESELLSCHAFT

SIEBZEHNTER BAND

1928

WALTER DE GRUYTER \& CO.

VORMIALS G. J.GÖSCHEN'SCHE VERLAGSIANDLUNG -J.GUT'TENTAG, VERLAGS. IBUCHHANDLUNG - GEORG REIMER - KARL J. 'TRÜBNER - VEIT \& COMP.

BERLIN UND LEIPZIG 


\section{Verheimlichung des Namens.}

\section{Von Ignaz Goldziher †.}

In einem mehrfach variierten Haditspruch läl3t man den Propheten die Lehre erteilen: Wenn ein Mann einem andern sich anbrüdert, so möge er ihn fragen nach seinem Namen, nach dem seines Vaters und welchem (Stamme) er angehört, denn dadurch wird die (gegenseitige) Liebe am ehesten fest geknüpft, $i d \bar{a} a \bar{a} \bar{a}$ ' $r$-rağulu ' $r$-raǵula' faijas'allu, 'an ismihi wa 'smi abīhi ua-mimman huua fa-innahu ausalu. li'l-maxadda (Ibn Sa'd VI 43,8; Tirmidi, Sahn̄h II 63, 4 v. u. Usd al-(xăba $\mathrm{V} 121,3$ v. u.). In einem andern Hadit, wird unter den drei Dingen, an denen man die Unzulänglichkeit eines Menschen erkennt, erwähnt, daß3 er jemandem begegnet, dessen Bekanntschaft ihm lieb ist, und sich von ihm trennt, ohne nach seinem Namen und seiner Abstammung gefragt zu haben (bei Gazali III 43, 19). Auch unter den von Zaid b. 'Alì gesammelten Lehren des 'Alī wird von diesem die Belehrung überliefert, daß es sechs Eigentümlichkeiten gebe, deren Betätigung ein Muslim dem andern schuldet; darunter: daß er ihm seinen und seines Vaters Namen sowie auch seinen Wohnort bekannt mache (Corpus Juris' di Z. b. 'A. ed. E. Griwini 275, 4).

Aus solchen Lehren zieht al-Sa'bì die Folgerung: Wenn ein Mann mit einem andern zusammentrifft und sagt: Ich kenne sein Antlitz, aber ich weiß nicht seinen Namen, so ist dies die Bekanntschaft von Blödsinnigen fi'r-raǵuli juǵălisu, 'r-raǵula fa-jakülu a'rifu. waǵhahu wa-lā a'rifu 'smahu fa-tilka ma'rifatu. 'n-naukā. (Ihjō II $165,1)$.

Solche Ermahnungen führen zur Folgerung, daß es unter den alten Arabern Sitte war, bei einer Begegnung, aber auch bei intimerem Verkehr, wenigstens bei Beginn desselben, nach dem Namen des Partners nicht zu fragen. Man legt sogar Wert darauf, seinen Namen zu verheimlichen oder zu verschleiern. Es kommt vor, daß vor dem Eingehen in eine Verhandlung zwischen den Parteien ein bindendes Ubbereinkommen geschlossen wird, da/3 sie gegenseitig ihre Namen

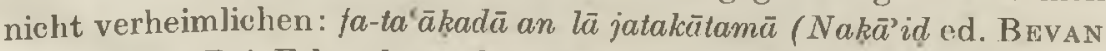
1024, 2 ff.). Bei Erkundung der Identität schejnt man höchstens so weit gegangen zu sein, mit Vermeidung der Neugier um den Personen-

1) In einer Version bei S uhrawardi, 'Awärif al-Ma'ärit, Kap. 54, IV, 73. wo die Ermahnung des Propheten anläßlich eines bestimmten Falles un Ibn "Omar gerichtet ist: ida ahbabta ahadan....

1 Islaul XYII 
namen des Gastes, nur nach dem Stamm zu fragen, dem die betreffende Person angehört (z. B. Agjān $\vec{\imath}$ VII 186. 17: mimmnn ar-raǵul, vgl. 1001 Nacht III 257, 8 v.u.).

Die Verheimlichung des Namens scheint, wie bei vielen Volkerschaften die Verhïllung des Antlitzes ${ }^{1}$ ). ursprünglich in der Besorgnis darüber begründet $z \|$ sein, sich durch Kenntnisgabe wegen einer verwirkten Blutschuld der Blutrache preiszugeben, was durch Verheimlichung der Identität vermieden werden sollte $\left.{ }^{2}\right)$. Der wegen einer Blutschuld Flïchtige kann mit Verschweigung seines Namens den Schutz eines \%eltes ansprechen, bei dessen Bewohnern er sich vor seinen Verfolgern verbergen möchte (Ağänì XXI, 78, 7 ağinä mutana kkiran). Der mustagia nennt seinen Namen. wenn der un Schutz Gehetene denselben mit Ausschluß einer bestimmten Person zu gewähren bereit ist. mit der jener nicht identisch ist (Mālik b. Asmā, Agāñ XVI 42, 15 11. 21). Auf die Frage man lakinm, ,Wer bist du?" antwortet der befragte Kaisite: ..Dieses Fragen kann dich ja nicht interessieren" lā häǵata laka fi's-su'äli 'an dälika, worauf der Befragendè entgegnet: ,Hat denn der Islam nicht alle Blutfehde und allen Hall ausgelöscht? ?“ D. h. durch den Islam ist ja die Besorgnis wegen der Blutrache getilgt

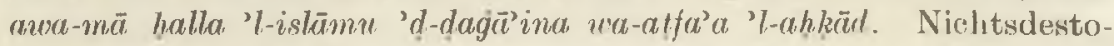
weniger verrät der Kaisite nicht mehr als den Namen der Sippe, der er innerhalh der Kaisitengruppe angehört ( Kä ä. Amälï I 117. 12ff.). Den eigenen Namen mag er nicht kundgeben (vgl. Ag̉āni IX 7, 15. 16). Dies geschicht jedoch bei Herausforderungen (muhäraza) zum Kampf (Hudail. I 69,7 ) und dient auch zur Bekundung der Fbenbiirtigkeit der Kämpfenden (Ibn Sacd II/I 10, I0ff.; 15. 17). Dem Herausfordernden wird in den Erzählungen dariber zumeist die formel in den Mund gegeben: ..Wer mich kennt, der keunt mich; wer mich nicht kennt (möge wissen) : ich bin N. der Sohn des N.", eine Formel die man literarisch auch auf andere (Gelegenheiten ïbertiagen hat ${ }^{3}$ ). Auch der

1) Dozy, Dictionnaire détaillé des noms des vêtements chez les Arabes 39yf. Hierher gehört auch die Verhüllung des Antlitzes des Abî Sufjūn b. al-Hārit und seines Sohnes, als sie vor dem Propheten erscheinen, Ihn Sacd IV/I 35) paenult.

2) Vgl. IAcou, Beduinenleben 40). Ther Verheimlichung des Numens нus undern Motiven s. Andraw, Ethnographische Parallelen (Stuttgat 1878) 179 ff.

3) Muhammed b. Ga'far. der 'alidische Prïtenrlent, heginnt mit clerselben. seine Entsagungserklürung ('Tabari III 994), obenso auch al-Aścarī, als er voun Katheder der Moschee in Basra seine Lossagung von dor Mu'tazila feierlich ankïnligt (Fihrist 181, 14); Auch den landstreichenden Bettler (mukuddT) läßt oine humoristische Darstellung dieselbe Formel gebrattchen i Bnihaki erl. SCHWALI, 624, 2). Vgl, auch die Selbstoinführung des unangemeldet in eine

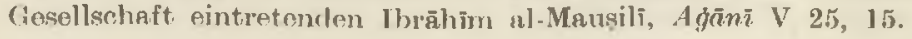


zum Schlage Ausholende gibt sich mit vollem Namen (oder der kunja, ZDMG XL 186, 5) zu erkennen (ḩudhā wa-anā 'bnu. . Ib n Hiśām 720, 2, Ibn Sacd IV/II 3, 17, Agjāni XII, 30, 8; 55, 7 v. u. ff. ; XV 74, 10 v. u.). Vgl. jedoch $A g \bar{a} n \bar{\imath}$ XVI, 55,12 v. u., wo Zaid al-Chail dem 'Āmir b. at-Ṭfail nur auf dessen Andrängen schließlich seinen Namen nennt.

Die Verheimlichung des Namens ${ }^{1}$ ) ist bis in späte Zeiten auf gesellschaftlichem Gebiete in Uhung geblieben. Unter südarabischen Beduinenstämmen, tribal custom ordains that no stranger shall be questioned even as regards his origin and purpose until he has been among you three whole days" (BURY, The Land of Uz 241). Und dieser Brauch reflektiert sich auch zuweilen an einigen Stellen der 1001 Nacht-Erzählungen. Freilich erfahren wir auch öfters das Gegenteil. Die einander Begegnenden nennen gegenseitig ihre Namen. Z. B. nur' in der einen 'Omal' an-No'män-Erzählung 390, 13; 392 paenult.; 393, 3 v. 11.; 400, 7: 413, 16:416, 5v. u. (der Būlāker Ausgabe 127!), im ersten Band). Jedoch auch dic Unbekumm(rtheit um den Namen kann beobachtet werden. Nachdem Gamil b. Ma'mal' drei Tage lang die Gastfreundschaft des jungen 'Udriten genossen hatte, fragt dieser erst am vierten 'Tag hei der Verabschiedung des (Yastes um dessen Namen und seine Stammesangehörigkeit (II1, 255). Der (Gatte der Zain al-Mawāsif (ein Jude) lebt bereits seit längerer Zeit, im vertrauten Verhältnis zu Masrūr, sie sind schon übereingekommen. miteinander in Handelsgenossenschaft zı treten, ohne daß3 jener sich um den Namen des F'reundes gekümmert hätte. Daß aber Masrū nasrānī sei, hat ihn früher interessiert als die Kenntnis seines Namens ${ }^{2}$ ), um den er sich. nach mehreren 'Tagen, auch nur aus Eifersucht bokiimmert. um herauszubekommen, ob er derselbe sei, den seine Crattin im Schlafe ruft (IV 110, 6 v. 11.; 111, 18).

1) Auch bei des Somuli wird der Fremde nicht nach seinem. Nanen gefragt: .. Die linge: Wer bist du ? drückt der Somali folgendermaßen aus: .. Du, welches

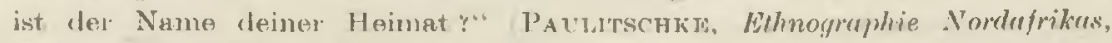
Berlin 1890, (15).

2) Vyl. \%1) 11 (: $x \times x I 1: 341$. 